\title{
Counting Triangle Crossings and Halving Planes*
}

\author{
T. K. Dey† and H. Edelsbrunner \\ Department of Computer Science, University of Illinois at Urbana-Champaign, \\ Urbana, IL 61801, USA \\ edels@cs.uiuc.edu
}

\begin{abstract}
Every collection of $t \geq 2 n^{2}$ triangles with a total of $n$ vertices in $\mathbb{R}^{3}$ has $\Omega\left(t^{4} / n^{6}\right)$ crossing pairs. This implies that one of their edges meets $\Omega\left(t^{3} / n^{6}\right)$ of the triangles. From this it follows that $n$ points in $\mathbb{R}^{3}$ have only $O\left(n^{8 / 3}\right)$ halving planes.
\end{abstract}

\section{Introduction}

Consider a set $S$ of $n$ points in three-dimensional Euclidean space, $\mathbb{R}^{3}$, and a collection of $t$ triangles with vertices in $S$. Clearly, if $t$ is not too large it could be that any two triangles intersect only in common vertices or edges, if at all. Indeed, this is possible for $t$ almost as large as $n^{2}$. Once $t$ exceeds $n^{2}$, some of the triangles start having common interior points, no matter how the points of $S$ are placed in space. If $t \geq \frac{3}{2} n^{2}$ we start to see crossing pairs, that is, pairs of vertex disjoint triangles with common interior points. This basic fact can be used to show that for $t \geq 2 n^{2}$ the number of crossing pairs is at least some positive constant times $t^{4} / n^{6}$. This extends a result of [1] that for $n$ points and $t \geq 4 n$ edges in $\mathbb{R}^{2}$ there are at least some positive constant times $t^{3} / n^{2}$ crossing edge pairs.

Why is this interesting? A consequence of the lower bound for crossing triangle pairs is that $n$ points in $\mathbb{R}^{3}$ have at most some positive constant times $n^{8 / 3} k$-sets, for any fixed $k, 0 \leq k \leq n$. A $k$-set of $S$ is a subset $S^{\prime}=S \cap H$, where $H$ is a half-space and $\left|S^{\prime}\right|=k$. For three-dimensional $k$-sets the first nontrivial bound of

\footnotetext{
* The research of $\mathbf{H}$. Edelsbrunner was supported by the National Science Foundation under Grant CCR-8921421 and under an Alan T. Waterman award, Grant CCR-9118874. Any opinions, findings and conclusions or recommendations expressed in this publication are those of the authors and do not necessarily reflect the view of the National Science Foundation.

† Current address: Department of Computer Science and Engineering, Indian Institute of Technology, Kharagpur, India 721302.
} 
$O\left(n^{3-1 / 343}\right)$ was reported in [4]. This was improved to $O\left(n^{8 / 3} \log ^{5 / 3} n\right)$ and later to $O\left(n^{8 / 3} \log ^{2 / 3} n\right)$ in [3] and [6]. This paper removes the remaining polylogarithmic factor from the upper bound. While the improvement is not dramatic, we feel the main contribution is the introduction of a new and more direct approach to proving bounds on $k$-sets.

\section{Crossing Triangles}

\section{Definitions and Results}

A set $V$ of three noncollinear points in $\mathbb{R}^{3}$ defines a triangle $\sigma_{V}=\operatorname{conv}(V)$. We say that two triangles have a nontrivial intersection if their intersection is neither empty nor a vertex or edge of both. Two triangles, $\sigma_{U}$ and $\sigma_{V}$, can form three different types of nontrivial intersections, as illustrated in Fig. 2.1. If $\sigma_{U} \cap \sigma_{V} \neq \varnothing$ and $U \cap V=\varnothing$, then we say that $\sigma_{U}$ and $\sigma_{V}$ cross. We also say that an edge crosses a triangle if it intersects the triangle without sharing a vertex with it. A finite point set is in general position if any $k$ of the points, $1 \leq k \leq 4$, are affinely independent. A set $S$ of $n$ points in general position in $\mathbb{R}^{3}$ defines a collection of $\left(\begin{array}{l}n \\ 3\end{array}\right)$ triangles, denoted $\left(\begin{array}{l}S \\ 3\end{array}\right)$. For any

$$
T \subseteq\left(\begin{array}{l}
S \\
3
\end{array}\right)
$$

we write $x(S, T)$ for the number of crossing triangle pairs in $T$. Furthermore,

$$
x(n, t)=\min _{|S|=n,|T|=t} x(S, T) .
$$

Similarly, we write $y(S, T)$ for the maximum number of triangles in $T$ crossed by a single edge of a triangle in $T$, and we set

$$
y(n, t)=\min _{|S|=n,|T|=t} y(S, T) .
$$

The results of this section are summarized in the following theorem.
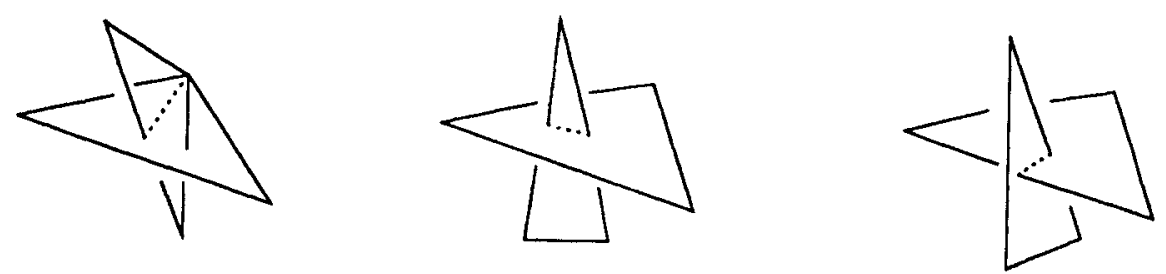

Fig. 2.1. The left two triangles have a nontrivial intersection but they do not cross. The triangles in the middle and to the right are vertex disjoint and cross. 
(2.1) Theorem. There are positive constants $c_{1}, c_{2}, c_{3}, c_{4}$ so that

$$
c_{1} \frac{t^{4}}{n^{6}}<x(n, t)<c_{2} \frac{t^{3}}{n^{3}}
$$

and

(ii)

$$
c_{3} \frac{t^{3}}{n^{6}}<y(n, t)<c_{4} \frac{t^{2}}{n^{3}}
$$

for

$$
2 n^{2} \leq t \leq\left(\begin{array}{l}
n \\
3
\end{array}\right)
$$

Proof of the Lower Bounds

We begin with a trivial but most useful observation.

(2.2) If triangles $\sigma$ and $\sigma^{\prime}$ have a nontrivial intersection, then there is an edge of one triangle that crosses the other triangle, and if $\sigma$ and $\sigma^{\prime}$ cross, then there are two such edges.

Notice that because of (2.2) the lower bound for $x(n, t)$ implies the lower bound for $y(n, t)$. To see this, count a crossing between two triangles once for each edge crossing the other triangle. Give one unit of credit to each such edge. There are two credits per crossing and at most $3 t$ edges. Assume the lower bound for $x(n, t)$ and use the pigeonhole principle to get an edge with more than $c_{3}\left(t^{3} / n^{6}\right)$ credits, where $c_{3}=2 c_{1} / 3$.

(2.3) Let $S$ be a set of $n$ points in general position in $\mathbb{R}^{3}$ and $T \subseteq\left(\begin{array}{l}S \\ 3\end{array}\right)$.

(i) $|T|<n^{2}$ if no two triangles in $T$ have a nontrivial intersection.

(ii) $|T|<\frac{3}{2} n^{2}$ if no two triangles in $T$ cross.

Proof. Consider a point $p_{i} \in S$ and all incident triangles in $T$. Let $\delta_{i}$ be the number of edges with endpoint $p_{i}$. Clearly, $\delta_{i} \leq n-1$ because there are only $n-1$ other endpoints. Intersect these triangles with a sphere around $p_{i}$ that is small enough so that each incident triangle intersects the sphere in a connected great-circle arc. Assuming the triangles form no nontrivial intersection, these arcs form a planar graph with $\delta_{i}$ vertices and hence fewer than $3 \delta_{i}$ arcs. It follows that $p_{i}$ is incident to fewer than $3 \delta_{i}$ triangles. Since each triangle is counted three times we have in total fewer than $\frac{1}{3} \sum_{p_{i} \in S} 3 \delta_{i}<n^{2}$ triangles. 
To prove (ii) assume that no two triangles in $T$ cross. An edge that crosses a triangle $\sigma$ can only belong to triangles that share a vertex with $\sigma$. There are at most three such triangles incident to that edge. Remove this edge together with the at most three triangles. Let $k$ be the number of such edges that need to be removed until no nontrivial intersection remains. We lose at most $3 k$ triangles and decrease the total vertex degree to $\sum_{p_{i} \in S} \delta_{i}-2 k$ or less. It follows that

$$
|T|<3 k+\left(\frac{1}{3} \sum_{p_{i} \in S} 3 \delta_{i}-2 k\right)=k+\sum_{p_{t} \in S} \delta_{i}<\left(\begin{array}{l}
n \\
2
\end{array}\right)+2\left(\begin{array}{l}
n \\
2
\end{array}\right)<\frac{3}{2} n^{2}
$$

is an upper bound on the number of crossing-free triangles.

The rest of the argument for the lower bound on $x(n, t)$ is inductive and is inspired by the proof of a similar result for line segments in [1]. Specifically, we prove the following inequality.

(2.4) There is a positive constant $c$ so that

$$
x(n, t)>c\left(\begin{array}{l}
n \\
6
\end{array}\right) \frac{t^{4}}{\left(\begin{array}{l}
n \\
3
\end{array}\right)^{4}} \quad \text { for } \quad 2 n^{2} \leq t \leq\left(\begin{array}{l}
n \\
3
\end{array}\right)
$$

Proof. Notice that we have $n \geq 15$ because

$$
2 n^{2} \leq t \leq\left(\begin{array}{l}
n \\
3
\end{array}\right)
$$

To cover the base case of the induction consider the range $2 n^{2} \leq t \leq \frac{5}{2} n^{2}$. Because of (2.3(ii)), the number of crossing pairs is at least $t-\frac{3}{2} n^{2}$. Furthermore,

$$
c\left(\begin{array}{l}
n \\
6
\end{array}\right) \frac{t^{4}}{\left(\begin{array}{l}
n \\
3
\end{array}\right)^{4}}<\frac{5^{4}}{2^{4}} \cdot \frac{3 !^{4}}{6 !} \cdot c \cdot \frac{n^{5}}{(n-1)^{3}} \leq \frac{625}{16} \cdot \frac{9}{5} \cdot \frac{15^{3}}{14^{3}} \cdot c n^{2}<\frac{n^{2}}{2} \leq t-\frac{3}{2} n^{2}
$$

as long as $c<\left(16 \cdot 5 \cdot 14^{3}\right) /\left(2 \cdot 625 \cdot 9 \cdot 15^{3}\right)$.

The inductive step assumes $t>\frac{5}{2} n^{2}$ and $x(S, T)=x(n, t)$. For a point $p_{i} \in S$, let $T_{i} \subseteq T$ contain all triangles not incident to $p_{i}$, and define $t_{i}=\left|T_{i}\right|$. For each crossing pair in $T$ count the vertices not incident to either triangle. The sum, over all crossing pairs, is $(n-6) \cdot x(S, T)$. Alternatively, we can think of this sum as counting the crossing pairs in $T_{i}$, for each $p_{i} \in S$. Therefore,

$$
(n-6) \cdot x(n, t)=(n-6) \cdot x(S, T)=\sum_{p \in S} x\left(S-\left\{p_{i}\right\}, T_{i}\right) \geq \sum_{p_{i} \in S} x\left(n-1, t_{i}\right)
$$


Since $p_{i}$ is incident to at most $\left(\begin{array}{c}n-1 \\ 2\end{array}\right)$ triangles, we have

$$
t_{i}>\frac{5}{2} n^{2}-\left(\begin{array}{c}
n-1 \\
2
\end{array}\right)>2(n-1)^{2}
$$

so we can apply the induction hypothesis to $S-\left\{p_{i}\right\}$ and $T_{i}$. This yields

$$
(n-6) \cdot x(n, t)>c \frac{\left(\begin{array}{c}
n-1 \\
6
\end{array}\right)}{\left(\begin{array}{c}
n-1 \\
3
\end{array}\right)^{4}} \sum_{p_{i} \in S} t_{i}^{4}
$$

Observe that $\sum_{p_{i} \in S} t_{i}=(n-3) t$, and that this implies $\sum_{p_{i} \in S} t_{i}^{4} \geq n((n-3) t / n)^{4}$. Therefore,

$$
x(n, t)>c \cdot \frac{n\left(\begin{array}{c}
n-1 \\
6
\end{array}\right)}{n-6} \cdot \frac{\left(\frac{n-3}{n}\right)^{4}}{\left(\begin{array}{c}
n-1 \\
3
\end{array}\right)^{4}} \cdot t^{4}=c\left(\begin{array}{l}
n \\
6
\end{array}\right) \frac{t^{4}}{\left(\begin{array}{l}
n \\
3
\end{array}\right)^{4}}
$$

Recall that $(2.4)$ holds for $c<\left(16 \cdot 5 \cdot 14^{3}\right) /\left(2 \cdot 625 \cdot 9 \cdot 15^{3}\right)$. So $c_{1}$ needs to be chosen so that

$$
c_{1} \frac{t^{4}}{n^{6}} \leq c\left(\begin{array}{l}
n \\
6
\end{array}\right) \frac{t^{4}}{\left(\begin{array}{l}
n \\
3
\end{array}\right)^{4}}<c \cdot \frac{3 !}{6 !} \cdot \frac{t^{4}}{n^{3}(n-1)^{3}} \leq c \cdot \frac{9}{5} \cdot \frac{15^{3}}{14^{3}} \cdot \frac{t^{4}}{n^{6}}
$$

Hence, the lower bounds of Theorem 2.1 hold for $c_{1}=\frac{1}{80}<16 /(2 \cdot 625)$ and $c_{3}=2 c_{1} / 3=\frac{1}{120}$.

\section{Proof of the Upper Bounds}

The upper bound for $y(n, t)$ in (ii) of Theorem (2.1) implies the upper bound for $x(n, t)$ in (i). To see this assume no edge crosses $c_{4}\left(t^{2} / n^{3}\right)$ or more triangles. Since there are at most $3 t$ edges the total credit accumulated is less than $3 c_{4}\left(t^{3} / n^{3}\right)$. Each crossing pair of triangles generates two credits which implies that there are fewer than $c_{2}\left(t^{3} / n^{3}\right)$ such pairs, where $c_{2}=3 c_{4} / 2$.

To prove the upper bound on $y(n, t)$ we choose the points of $S$ on the moment curve $M(\tau)=\left(\tau, \tau^{2}, \tau^{3}\right)$ defined for all $\tau \in \mathbb{R}$. For two points $a=M\left(\tau_{1}\right)$ and 
$b=M\left(\tau_{2}\right)$ we write $a \prec b$ if $\tau_{1}<\tau_{2}$. It is easy to establish that $M$ intersects any plane in at most three points.

(2.5) Let $a \prec b \prec c$ and $p \prec q$ be five different points on the moment curve in $\mathbb{R}^{3}$. The edge $p q$ crosses the triangle abc iff $a \prec p \prec b \prec q \prec c$.

Proof. We first show that if the five points do not lie in the sequence $a \prec p \prec b \prec q \prec c$, then $p q$ does not cross $a b c$. Assume without loss of generality that neither $p$ nor $q$ lie between $a$ and $b$ and suppose $p q$ crosses $a b c$. Move $b$ continuously on $M$ toward $a$. At some moment before $a b c$ shrinks to a line segment, $p q$ intersects an edge of $a b c$. At this moment, the four endpoints of the two edges are coplanar, which contradicts the fact that $M$ intersects every plane in at most three points.

Next, we prove that if $a \prec p \prec b \prec q \prec c$, then $p q$ crosses $a b c$. By Randon's theorem [8] the set $U=\{a, b, c, p, q\}$ can be partitioned into $U=V \dot{\cup} W$ so that $\operatorname{conv}(V) \cap \operatorname{conv}(W) \neq \varnothing$. Since all five points are vertices of $\operatorname{conv}(U)$, the partition must be into two and three points. All cases other than $V=\{p, q\}$ and $W=\{a, b, c\}$ are excluded by the above argument.

Consider the set $S=\left\{p_{i}=M(i) \mid 1 \leq i \leq n\right\}$, with $n \geq 15$. Define the width of a triangle $\sigma=p_{i} p_{j} p_{k}$, with $i<j<k$, equal to $\min \{j-i, k-j\}$. For an integer $w$, let $T_{w}$ be the collection of triangles of width $w$ or less. The number of triangles in $T_{w}$ can be counted by taking all index pairs $i, j$, with $j-i \leq w$, and joining them with an arbitrary third index. Each triplet is counted at most three times. Therefore,

$$
\begin{aligned}
\left|T_{w}\right| & >\frac{1}{3}(n-2) \sum_{\ell=n-w}^{n-1} \ell \\
& =\frac{n-2}{6}(2 n w-w(w+1)) .
\end{aligned}
$$

We use $n \geq w+1$ and $(n-2) / n \geq \frac{13}{15}$, and then $w \geq(w+1) / 2$ to get

$$
\begin{aligned}
\left|T_{w}\right| & >\frac{13}{6 \cdot 15} n^{2} w \\
& >\frac{1}{14} n^{2}(w+1)
\end{aligned}
$$

The triangle $\sigma=p_{i} p_{j} p_{k}$ crosses a fixed edge $p_{\ell} p_{m}$, with $\ell<m$, iff $i<\ell<j<m<k$. The number of such triangles $\sigma \in T_{w}$ is less than

$$
2\left(\begin{array}{l}
w \\
2
\end{array}\right) n<w^{2} n
$$


For a given $t$, choose $w$ so that $\left|T_{w-1}\right|<t \leq\left|T_{w}\right|$ and let $T \subseteq T_{w}$ be arbitrary with $|T|=t$. Then

$$
y(n, t) \leq y(S, T)<w^{2} n=\frac{\left(\frac{1}{14} n^{2} w\right)^{2}}{\frac{1}{196} n^{3}}<196 \frac{\left|T_{w-1}\right|^{2}}{n^{3}}<196 \frac{t^{2}}{n^{3}} .
$$

This establishes the upper bounds of Theorem (2.1) with $c_{4}=196$ and $c_{2}=294$.

Remark. Let us compare the upper bound in (i) of Theorem (2.1) with the corresponding lower bound. Both bounds are valid for

$$
2 n^{2} \leq t \leq\left(\begin{array}{l}
n \\
3
\end{array}\right)
$$

For $t=\left|T_{1}\right|=(n-2)^{2}$ we can find $n$ points and $t$ triangles, namely, the set $T_{1}$, so that no two triangles have a nontrivial intersection. Hence, $x\left(n,(n-2)^{2}\right)=0$ although $x\left(n, 2 n^{2}\right)>c_{1}\left(\left(2 n^{2}\right)^{4} / n^{6}\right)>\frac{1}{5} n^{2}$. So as $t$ increases from $(n-2)^{2}$ to $2 n^{2}$, the minimum number of crossing pairs goes up from 0 to quadratic in $n$. The upper bound for $t=2 n^{2}$ is $c_{2}\left(\left(2 n^{2}\right)^{3} / n^{3}\right)=2352 n^{3}$. This suggests the possibility that $x(n, t)$ is indeed cubic in $n$ for this choice of $t$. Suppose this is indeed the case. Using the same induction as in the proof of (2.4), we could then prove that $x(n, t)$ is proportional to $t^{3} / n^{3}$, for all

$$
2 n^{2} \leq t \leq\left(\begin{array}{l}
n \\
3
\end{array}\right)
$$

As explained in Section 3, this would imply that $n$ points in $\mathbb{R}^{3}$ have only $O\left(n^{5 / 2}\right)$ halving planes.

\section{Halving Planes}

Let $S$ be a set of $n$ points in $\mathbb{R}^{3}$. Assume that $n$ is odd and that no four points are coplanar. For three points $a, b, c \in S$ let $\sigma=a b c$ be the corresponding triangle and let $h$ be the plane that contains $a, b, c$. We call $h$ a halving plane, and $\sigma$ a halving triangle, if there are $(n-3) / 2$ points on each side of $h$. The following extension of the two-dimensional Lovász lemma [7] has been used in [3] and [4]. We say a line crosses a triangle if it intersects the triangle but not any of its edges.

(3.1) A line crosses less than $n^{2} / 8$ halving triangles.

Proof. Consider a plane that contains no point of $S$, and let $l_{0}$ and $l_{1}$ be two parallel lines in this plane so that $l_{1} \cap \operatorname{conv}(S)=\varnothing$. For each $0 \leq \tau \leq 1$ define $l_{\tau}=\tau l_{0}+(1-\tau) l_{1}$. As $\tau$ increases from 0 to $1, l_{\tau}$ translates from $l_{1}$ to $l_{0}$. The 
number of halving triangles crossed by $l_{\tau}$ changes only when $l_{t}$ translates through an edge,

$$
a b \in\left(\begin{array}{l}
S \\
2
\end{array}\right) \text {. }
$$

A simple rotation argument shows that the number increases or decreases by at most one. Given $l_{0}$ we can choose $l_{1}$ so that $l_{\tau}$ translates through fewer than $\frac{1}{8} n^{2}$ edges as follows. Choose the plane through $l_{0}$ so that there are $(n-1) / 2$ and $(n+1) / 2$ points on the two sides. There are $\left(n^{2}-1\right) / 4$ edges $a b$ that cross the plane and thus there is one side of $l_{0}$ with fewer than $n^{2} / 8$ edges. It follows that $l_{0}$ crosses at most $n^{2} / 8$ halving triangles.

We prove an upper bound on the number of halving planes by selecting a line that crosses many triangles. Suppose there are $t$ halving triangles. Condition (ii) of Theorem (2.1) implies that there is a line that crosses more than $c_{3}\left(t^{3} / n^{6}\right)$ of them, with $c_{3}=\frac{1}{120}$. Because of $(3.1), c_{3}\left(t^{3} / n^{6}\right)<n^{2} / 8$ which implies the main result of this section.

(3.2) Theorem. The number of halving planes defined by $n$ points in $\mathbb{R}^{3}$ is less than $c_{5} n^{8 / 3}$.

The constant, $c_{5}$, can be chosen equal to $1 /\left(8 c_{3}\right)^{1 / 3}=15^{1 / 3}<3$.

Remarks. A subset $S^{\prime}$ of $S$ is a $k$-set if $\left|S^{\prime}\right|=k$ and $S^{\prime}=S \cap H$ for some half-space $H$. The number of halving planes is closely related to the number of $k$-sets of $S$. Indeed, the number of $k$-sets, for $k=(n-1) / 2$, does not exceed three times the number of halving planes, see [5]. For other values of $k$ consider the planes spanned by three points of $S$ so that $k-1$ points lie on one and $n-k-2$ points lie on the other side. Again, the number of such planes is proportional to the number of $k$-sets. Furthermore, the same arguments imply the same upper bound as in Theorem (3.2) for any fixed $k$.

The bound in Theorem (3.2) slightly improves the ones in [3] and [6]. Besides this improvement, the main contribution of the new proof is the uncovering of the close relationship between $k$-sets and the combinatorics of crossings between edges and triangles in $\mathbb{R}^{3}$.

\section{Discussion}

Maybe the most interesting extension of the methods in this paper is to four and higher dimensions. It is possible to generalize all results of Section 2 to crossing pairs of $(d-1)$-simplices in $\mathbb{R}^{d}$. Specifically, we can show that $t \geq c n^{d-1}(d-1)$ simplices defined by $n$ points in $\mathbb{R}^{d}$ form at least $c^{\prime}\left(t^{d+1} / n^{d(d-1)}\right)$ crossing pairs. However, to prove a nontrivial upper bound on the number of halving hyper- 
planes, we need a nontrivial lower bound on the number of $(d-1)$-tuples of $(d-1)$-simplices that have a common point in their interiors. While the main part of the induction in (2.4) generalizes to this situation, we have no good extensions of the base case in (2.3).

We should be more accurate. Recent results obtained with methods from algebraic topology [9] do imply a nontrivial base case, and induction can be used to get nontrivial bounds for $(d-1)$-tuples of $(d-1)$-simplices and for halving hyperplanes. These bounds come out very similar to those derived in [2] and are therefore omitted. An interesting question is whether the elementary methods of this paper suffice to derive such nontrivial bounds.

\section{References}

1. M. Ajtai, V. Chvátal, M. M. Newborn, and E. Szemerédi. Crossing-free subgraphs. Ann. Discrete Math. 12 (1982), 9-12.

2. N. Alon, I. Bárány, Z. Füredi, and D. J. Kleitman. Point selection and weak $\varepsilon$-nets for convex hulls. Combin. Probab. Comput. 1 (1992), 189 200.

3. B. Aronov, B. Chazelle, H. Edelsbrunner, L. J. Guibas, M. Sharir, and R. Wenger. Points and triangles in the plane and halving planes in space. Discrete Comput. Geom. 6 (1991), 435-442.

4. I. Bárány, Z. Füredi, and L. Lovász. On the number of halving planes. Proc. Sth Ann. Symp. on Computational Geometry, 1989, pp. 140-144.

5. H. Edelsbrunner. Algorithms in Combinatorial Geometry. Springer-Verlag, Heidelberg, 1987.

6. D. Eppstein. Improved bounds for intersecting triangles and halving planes. J. Combin. Theory Ser. A 62 (1993), 176-182.

7. P. Erdős, L. Lovász, A. Simmons, and E. G. Straus. Dissection graphs of planar point sets. In A Survey of Combinatorial Theory, J. N. Srivastava et al., eds., pp. 139-149, North-Holland, Amsterdam, 1973.

8. J. Radon. Mengen konvexer Körper, die einen gemeinsamen Punkt enthalten. Math. Ann. 83 (1921), 113-115.

9. R. Živaljević and S. Vrećica. The colored Tverberg's problem and complexes of injective functions. J. Combin. Theory Ser. A 61 (1992), 309-318.

Received December 3, 1992, and in revised form January 29, 1994. 